livraisons

d'Histoire

de l'Architecture

\section{Livraisons de l'histoire de l'architecture}

$13 \mid 2007$

Architectures des établissements d'enseignement supérieur

\title{
Le collège Sainte-Barbe de Paris : des frères Labrouste à Ernest Lheureux
}

The College Sainte-Barbe in Paris: from the Labrouste brothers to Ernest

Lheureux

Das Collège Sainte-Barbe in Paris : von den Gebrüdern Labrouste bis Ernest

Lheureux

\section{Annabelle Lebarbé}

\section{OpenEdition \\ Journals}

Édition électronique

URL : http://journals.openedition.org//ha/418

DOI : $10.4000 /$ lha. 418

ISSN : $1960-5994$

Éditeur

Association Livraisons d'histoire de l'architecture - LHA

Édition imprimée

Date de publication : 10 juin 2007

Pagination : 137-148

ISSN : 1627-4970

Référence électronique

Annabelle Lebarbé, "Le collège Sainte-Barbe de Paris : des frères Labrouste à Ernest Lheureux ", Livraisons de l'histoire de l'architecture [En ligne], 13 | 2007, mis en ligne le 10 juin 2009, consulté le 19 avril 2019. URL : http://journals.openedition.org//ha/418 ; DOI : 10.4000//ha.418

Ce document a été généré automatiquement le 19 avril 2019

Tous droits réservés à l'Association LHA 


\section{Le collège Sainte-Barbe de Paris : des frères Labrouste à Ernest Lheureux}

The College Sainte-Barbe in Paris : from the Labrouste brothers to Ernest

\section{Lheureux}

Das Collège Sainte-Barbe in Paris : von den Gebrüdern Labrouste bis Ernest

Lheureux

\section{Annabelle Lebarbé}

Situé au cœur du quartier latin sur la montagne Sainte-Geneviève, le collège Sainte-Barbe ouvre ses portes en 1460 en qualité de pension pour les élèves qui suivent des cours dans les collèges voisins ${ }^{1}$. Bordé par les rues des Chiens, des Cholets, de Reims et des SeptVoies, le collège est situé sur un terrain exigu, en pente et il est composé de maisons accolées appartenant à différents propriétaires. Au mois d'avril 1793, le collège est obligé de fermer ses portes; les bâtiments sont réquisitionnés comme biens nationaux et attribués au collège de l'Égalité qui devient le Prytanée français. Parallèlement, la Convention crée en 1795 les écoles centrales, prélude à la création des futurs lycées. À Paris, c'est dans l'ancienne abbaye de Sainte-Geneviève que l'on installe l'école centrale du Panthéon, actuel lycée Henri IV. Sainte-Barbe rouvre cependant en 1798 grâce à Victor de Lanneau, et ses successeurs engagent des reconstructions : la première commence en 1840 par le collège classique ; la seconde s'achève en 1884 par la fin des travaux de l'école préparatoire. Le collège Sainte-Barbe a fermé ses portes en 1997 mais une partie de ces bâtiments est encore visible, notamment les deux entrées : l'une correspond à l'entrée de la bibliothèque Cujas dans la rue du même nom (ancienne rue Saint-Étienne-des-Grès) ; la seconde, située rue Valette (ancienne rue des Sept-Voies), servira d'entrée à la future bibliothèque inter-universitaire Sainte-Barbe.

Nous nous proposons d'étudier ici ces deux projets scolaires architecturaux, l'exemple de Sainte-Barbe se révélant particulièrement intéressant à une période où se définit peu à peu une politique en faveur de l'enseignement secondaire. Institution privée non 
confessionnelle, Sainte-Barbe dispose d'une grande liberté pour mettre en œuvre ses propres solutions innovantes en matière d'architecture scolaire.

2 L'histoire générale du collège Sainte-Barbe est connue grâce aux travaux de Jules Quicherat ${ }^{2}$ et Georges Nouvel ${ }^{3}$. Nous avons pu en renouveler l'étude grâce notamment aux archives du collège conservées aux archives départementales de Paris ${ }^{4}$, à la révision cadastrale de $1876^{5}$, à la correspondance et aux notes d'Alexandre Labrouste adressées à Jules Quicherat ${ }^{6}$ et aux publications des revues d'architecture au moment de la reconstruction de l'institution?.

\section{La renaissance du collège}

3 Sainte-Barbe renaît de ses cendres grâce à l'initiative de Victor de Lanneau, directeuradjoint du Prytanée depuis 1797, qui devient locataire en son nom des différents immeubles de la rue des Sept-Voies qui formaient le collège et ont été vendus comme biens nationaux ${ }^{8}$. Il inaugure la reprise des études le jour de la fête de la sainte patronne, le 4 décembre 1798, et fait du collège un établissement moderne. La loi Fourcroy du 11 floréal an X ( $1^{\mathrm{er}}$ mai 1802) créé les lycées; les établissements privés peuvent cependant subsister moyennant un impôt payé à l'Université à partir de 1808. Si le collège donne quelques cours, il est avant tout une pension pour le secondaire. Le décret du 15 novembre 1815 limite en effet les initiatives privées, en prescrivant que «les élèves des institutions particulières établies dans les villes dotées d'un collège ou d'un lycée sont astreints à en suivre les enseignements $»^{9}$.

4 À partir de 1831, le collège Sainte-Barbe est confronté à ses dettes vis-à-vis du ministère et des propriétaires. L'État accorde un délai pour payer la rétribution universitaire, mais les particuliers sont sur le point de saisir le collège. Pour la deuxième fois de son histoire, Sainte-Barbe trouve son " sauveur $»^{10}$, en la personne de Claude Bellaigue, alors député de l'Yonne et ancien élève, qui propose de former une société en commandite avec ses anciens camarades ${ }^{11}$. La société de Sainte-Barbe devient propriétaire du collège le 30 décembre 1831. Quand Alexandre Labrouste est appelé au poste de directeur, le 3 avril 1838, l'institution se trouve relevée financièrement.

\section{La reconstruction du collège par les frères Labrouste}

\section{Une œuvre barbiste (ill. 1 et 2)}

5 Les bâtiments n'ont bénéficié d'aucuns travaux de grande ampleur depuis trois siècles ${ }^{12}$. 


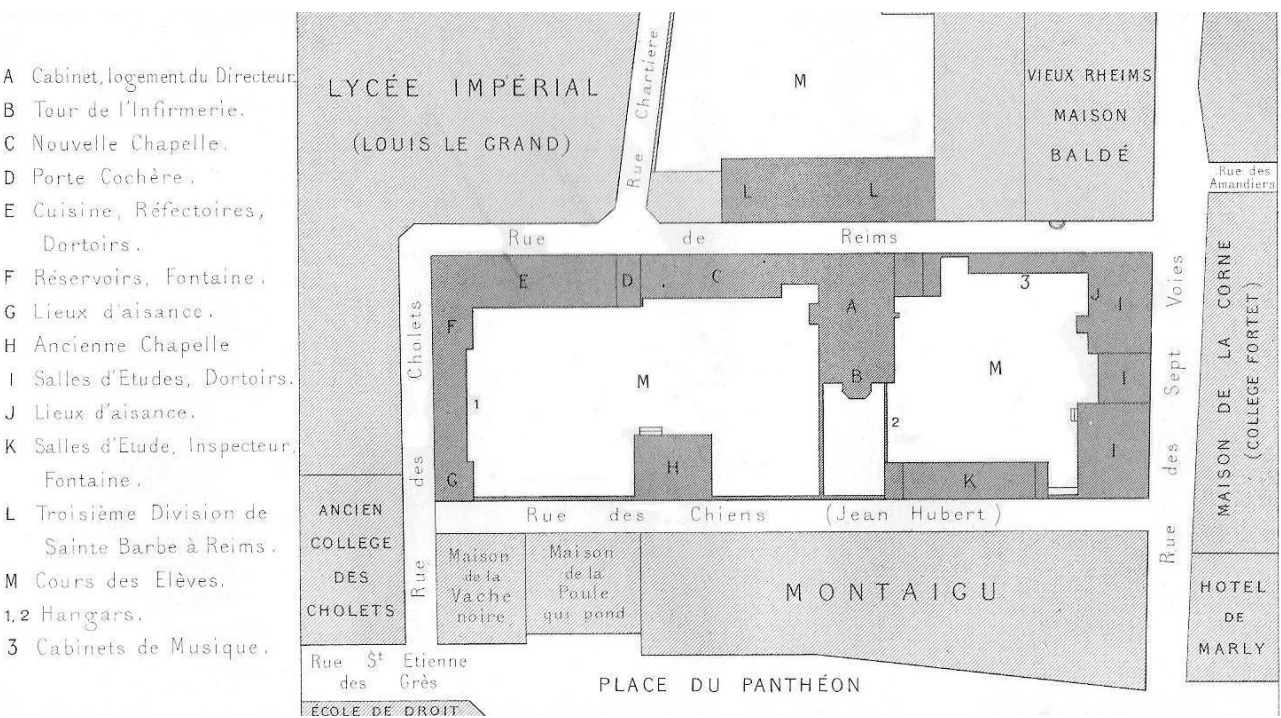

Jules Quicherat, Histoire de Sainte-Barbe, collège, communauté, institution, Paris, Hachette, 1860-1864, vol. 3, p. 25

Cl. A. Lebarbé

\section{2 : Plan de Sainte-Barbe en 1847}

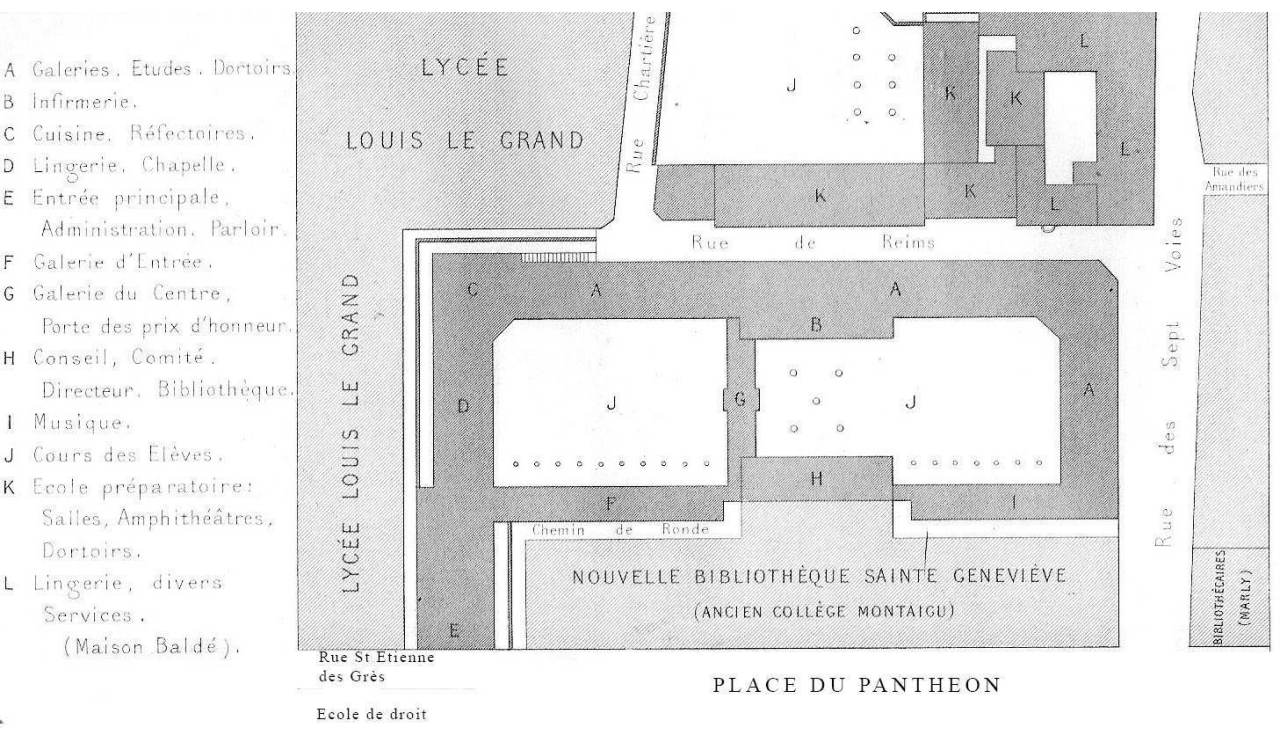

Jules Quicherat, Histoire de Sainte-Barbe, collège, communauté, institution, Paris, Hachette, 1860-1864, vol. 3, p. 257

Cl. A. Lebarbé

Deux événements vont permettre au directeur d'engager le chantier : un des baux expire en 1839, et, la même année, une poutre maîtresse craque dans un bâtiment de la rue des Sept-Voies ${ }^{13}$. Il profite de cet incident pour attaquer le propriétaire de la maison qui refuse de reconstruire. Selon Alexandre Labrouste :

Depuis quarante ans, on n'a fait aucuns travaux de consolidation, malgré les avertissements donnés au propriétaire. Il a fallu que la police, provoquée par la rumeur publique et l'alarme que l'apparence menaçante de maisons, surtout du côté de la rue, avait répandue dans le quartier, il a fallu que la police intervînt, fît 
étayer elle-même et lançât sommations pour que le propriétaire consentit à s'occuper de la propriété autrement que pour en toucher très exactement les loyers ${ }^{14}$.

7 Après un difficile procès ${ }^{15}$, le propriétaire permet au collège d'acquérir tout le terrain. Le collège ne dispose cependant pas des fonds nécessaires pour effectuer les travaux et doit faire le choix entre la dissolution de l'établissement, c'est-à-dire « renoncer presque à ce culte de dévouement et d'amitié si bienfaisant pour nous et nos enfants» ou bien «faire de nouveau un appel aux sentiments barbistes, former une société puissante, régulière, pouvant fournir les capitaux nécessaires pour acheter les immeubles où fleurit le collège depuis plus de quatre siècles, et édifier les bâtiments convenables à une grande institution $»^{16}$. La société fondée en 1831 est transformée en société anonyme, par l'ordonnance royale du 17 mars 1841. Des actions sont mises en ventes et le capital social est augmenté, ce qui permet à cinq cent quatre-vingts autres anciens élèves de devenir actionnaires ${ }^{17}$. L'association Sainte-Barbe est ainsi la première société commerciale à devenir propriétaire d'une maison d'éducation. La société signe des promesses d'achats sur les maisons qui forment le collège. Elle peut ainsi détruire les bâtiments et poser la première pierre, le 8 août 1840, de l'aile qui fait le retour de la rue des Sept-Voies sur la rue de Reims.

8 De même que seuls les anciens élèves ou parents d'anciens élèves peuvent souscrire à la société, les bâtiments du collège classique sont reconstruits par des anciens barbistes, car «chez nous, tout est fait par et pour les barbistes $»^{18}$ : Amaury Duval, le peintre de la chapelle, Augustin Dumont, le sculpteur chargé d'exécuter la statue de sainte Barbe, sont d'anciens élèves, ainsi que les entrepreneurs qui « sont père et oncle de quatre barbistes modernes $\aleph^{19}$. Quant aux architectes choisis par le directeur Alexandre Labrouste et le conseil d'administration, ils ne sont autres que les frères du directeur, Henri et Théodore, eux-mêmes anciens barbistes.

Dans son histoire de Sainte-Barbe, Quicherat ne mentionne qu'Henri Labrouste comme architecte de Sainte-Barbe. Or, Théodore et Henri sont cités tous deux comme architectes dans les comptes rendus des conseils d'administration jusqu'en 1842. Il semble qu'Henri ait laissé par la suite son frère s'occuper du chantier de Sainte-Barbe lorsque lui-même commence le chantier de la bibliothèque Sainte-Geneviève ${ }^{20}$. Henri aurait ainsi contribué à l'édification de la première partie du collège, mais seul Théodore aurait réalisé l'entrée sur la rue Cujas.

\section{UN PRÉCURSEUR EN MATIÈRE D'ARCHITECTURE SCOLAIRE}

Les lycées créés par la loi de 1802 se sont installés dans des bâtiments non destinés à cet usage et qui doivent être entretenus aux frais des communes. La législation sur les bâtiments de l'enseignement public, à laquelle Sainte-Barbe n'est pas contraint, n'apparaît qu'en 1843 sous l'impulsion du ministre Abel Villemain. La séparation par catégorie d'âge (petits, moyens et grands) est prescrite et doit être visible dans le plan du collège. Il y aura alors trois quartiers pour le préau, la cour, les salles de classes et d'études, les dortoirs et, en théorie, le réfectoire ${ }^{21}$. Ces principes sont déjà appliqués à Sainte-Barbe, qui s'inscrit comme précurseur en matière d'architecture scolaire et s'inspire des principes généraux des pédagogues et hygiénistes de son temps. SainteBarbe utilise déjà ces principes pour la réalisation des plans du collège dont la première pierre est posée le 8 août 1840. En effet, Saint-Louis est construit en 1820, Louis-le-Grand 
est reconstruit entre 1841 et 1881 . Quant au premier établissement construit de toute pièce, il s'agit de Chaptal dont les travaux s'achèvent en $1876^{22}$.

\section{LA FAÇADE}

11 Depuis sa création, Sainte-Barbe cherche à avoir une façade sur la place du Panthéon. Les terrains de l'ancien collège de Montaigu sont libres lorsque le collège commence ses travaux. Après de longues tractations avec la mairie de Paris, le collège obtient seulement d'annexer une partie de la rue des Chiens, afin de s'assurer «l'accès du collège par la meilleure avenue du Quartier Latin $»^{23}$.

12 L' « étroite mais élégante et sévère $»^{24}$ façade de la rue Saint-Étienne des Grès porte audessus de son entrée l'inscription "Sainte-Barbe » en lettres d'or, surmontée de l'étoile barbiste, et d'un écusson où la palme universitaire, " en signe d'alliance avec notre mère l'Université $»^{25}$ encadre les trois dates importantes du collège : 1460,1798 et 1841. La façade du collège et celle de la bibliothèque Sainte-Geneviève qui est alors édifiée sur les terrains de Montaigu s'harmonisent grâce à une frise végétale similaire en pierre audessus des fenêtres du rez-de-chaussée. Le directeur de Sainte-Barbe justifie cette harmonie de la sorte :

Quant à notre façade sur la place du Panthéon, nous l'avons mise, quant aux lignes principales et aux principaux motifs, en harmonie avec le monument de la nouvelle bibliothèque. Nous avons été construit avant elle ${ }^{26}$; la main de Labrouste, architecte de la bibliothèque, avait bien pu communiquer son projet non encore exécuté, au Labrouste, architecte du collège, sur la demande du Labrouste, directeur de SainteBarbe. Avouons cette confidence toute fraternelle pour que l'on ne se figure pas que c'est l'État qui a copié l'édifice barbiste. Il est tout simple que les deux monuments eussent un air de famille. Quoique nous n'eussions qu'une langue étroite, comme un simple cap, sur la place du Panthéon, nous avons pu cependant nous donner une entrée d'apparence assez monumentale. Il fallait, que l'on comprît tout de suite que ce n'était pas une maison ordinaire ${ }^{27}$.

\section{LE PLAN DU COLLÈGE (ILL. 3)}

Le collège construit par les frères Labrouste forme un U qui est calé sur la bibliothèque Sainte-Geneviève, ce qui le rend clos. 
III. 3 : Vue en perspective cavalière du collège classique et de l'école préparatoire

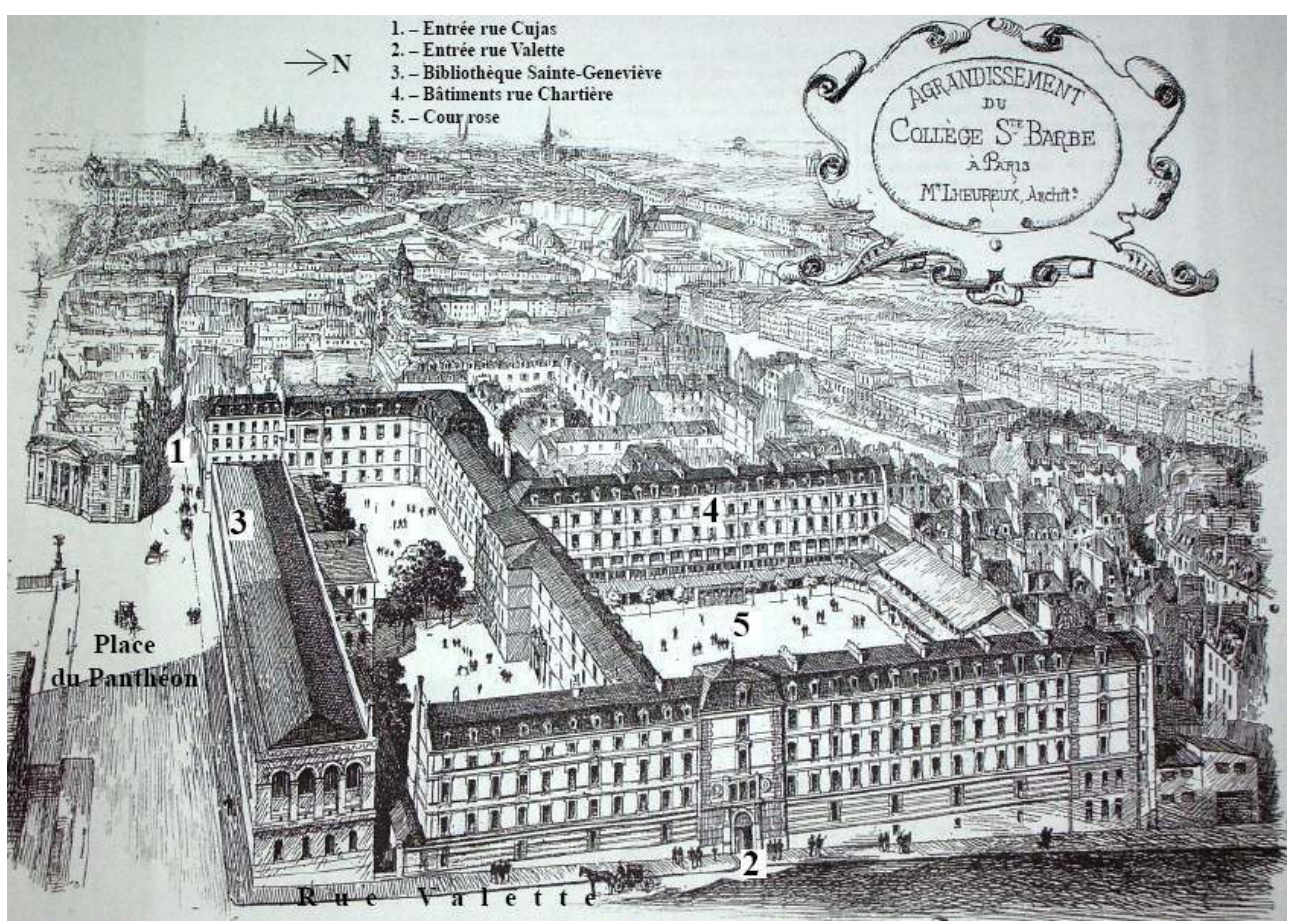

Montmory, «Collège Sainte-Barbe », La Semaine des constructeurs, 21 mars 1891, $2^{\mathrm{e}}$ série, $5^{\mathrm{e}}$ année, p. 463

Cl. A. Lebarbé

14 Le plan du rez-de-chaussée du collège est connu par une gravure d'Ernest Lheureux, l'architecte qui a refait l'école préparatoire quarante années après, par le cadastre ${ }^{28}$ et par un schéma de Quicherat. L'aile qui suit le cours de l'ancienne rue des Cholets abrite l'administration et au premier étage, les bureaux du directeur. À sa suite, l'aile ouest abrite la lingerie au sous-sol et la chapelle au premier étage, réalisées par Théodore Labrouste. Les réfectoires et les cuisines sont à l'angle de l'ancienne rue des Cholets et de l'aile qui longe la rue de Reims. Les études et les salles de cours occupent sûrement le rezde-chaussée et le premier étage des deux autres ailes, et les dortoirs seraient au second ou au troisième étage. Le petit et le moyen collège sont hermétiquement fermés ; seule la chapelle est commune aux deux. La chapelle de l'établissement n'est pas visible de l'extérieur comme c'était déjà le cas dans les anciens bâtiments. Sur la stalle du directeur, Alexandre Labrouste demande à son frère de graver «Ego Pro eis Rogo » ${ }^{29}$ et sur le prieDieu de la stalle, le directeur demande à ce que soit placé «le marteau même de M. de Lanneau, le marteau qui lui servait, et qui me sert à indiquer les mouvements debout, assis, à genoux $\rrbracket^{30}$. Les peintures de la coupole et de la corniche ont été réalisées par Amaury Duval. Le plafond est parsemé d'étoiles dans des caissons en trompe-l'œil. Sur les côtés, trois tableaux représentent les trois vertus théologales, la foi, l'espérance et la charité. Dans le chœur, en haut de la coupole, autour de la lanterne vitrée, court l'inscription « Hoc est mandatum meum ut deligatis invicem sicut ego dilegi vos ${ }^{31}$. 


\section{L'école préparatoire construite par Ernest Lheureux}

\section{Les travaux}

Sainte-Barbe décide d'ouvrir pour la rentrée de 1835 une classe de préparation aux grandes écoles, installée dans les locaux de l'ancien collège de Reims. Le succès est rapide : en 1853, plus du tiers de la promotion reçue à Polytechnique est pensionnaire à Sainte-Barbe. Sainte-Barbe doit cependant engager des travaux pour remédier au problème du logement des élèves. Un ancien élève d'Henri Labrouste, Ernest Lheureux, propose des plans pour l'école préparatoire, incluant un aménagement des voies, afin de la réunir au collège. Les rues de Reims, du Four et d'Écosse ${ }^{32}$ sont supprimées. À l'assemblée générale, le secrétaire général vante les mérites des travaux :

Notre École préparatoire, au point de vue de son local, de son installation matérielle, était à peu près restée jusqu'ici ce qu'elle était il y a trente ou quarante ans, c'est-à-dire sombre, étroite, triste. Aujourd'hui que le bien être matériel est la première loi de l'existence, il faut convenir que la vue de notre École préparatoire n'avait rien de précisément attrayant. Le luxe de nos rivaux leur a fait, plus d'une fois, donner la préférence. Nous avons tenu à les battre même sur ce terrain ${ }^{33}$.

Les démolitions commencent en 1881, et à la rentrée de 1882, les élèves peuvent entrer par la rue Valette ${ }^{34}$. L'année suivante, les locaux sont inaugurés. Lheureux est récompensé à l'exposition des projets d'établissements scolaires et la presse spécialisée loue sa réalisation. Pour Le Génie civil, «cette construction est une de celles qui représentent le mieux les derniers perfectionnements de l'art scolaire : mobiliers de classe confortables, dortoirs aménagés d'une façon pratique, salle de dessin spacieuse et monumentale $»^{35}$.

\section{LA FAÇADE}

La façade principale de l'école préparatoire s'étend sur la rue Valette et s'aligne avec le corps du bâtiment du collège classique en prolongeant celui-ci jusqu'à l'ancien $n^{\circ} 8$ de la rue des Sept-Voies. L'entrée est située en son milieu.

Les moyens financiers dont dispose l'architecte sont assez faibles, mais il use de moyens décoratifs peu coûteux pour rendre la façade attrayante. Il joue avec les couleurs des matériaux, la brique rouge de Bourgogne, la meulière grise et la pierre de taille blanche. La sobriété du portail en fer, orné du nom de Sainte-Barbe, contraste avec le décor qui l'encadre. Au milieu de l'arche de la porte, un livre ouvert indique les quatre dates importantes de l'histoire du collège: les mêmes que celles indiquées sur la rue Cujas $(1460,1798,1841)$, suivies de 1883, année de l'inauguration de cette aile sur la rue Valette. De part et d'autre de l'arche, deux colonnes renforcent la monumentalité de l'entrée. Deux lions ornent le bas des colonnes. Le premier étage au-dessus de l'entrée est composé d'un module de trois baies ponctuées de pilastres et encadrées par deux médaillons commémoratifs représentant Victor de Lanneau et Alexandre Labrouste. Il anticipe ainsi sur une circulaire ministérielle de 1865 selon laquelle «l'aspect des lycées devrait être simple et gai » et « la demeure des enfants doit être riante $»^{36}$. 


\section{LA « COUR ROSE »}

19 La «cour rose » de l'école préparatoire, surnommée ainsi à cause de la couleur de ses briques, est limitée au sud par le collège classique et au nord par un gymnase d'étée ${ }^{37}$. Les deux bâtiments qui longent la rue Valette et l'impasse Chartière comptent six niveaux et leurs façades sont symétriques. La forte déclivité du terrain a permis une utilisation des sous-sols. Conçus en briques avec de grandes baies vitrées, les sous-sols contiennent le réfectoire et les cuisines du côté de l'impasse Chartière, et les salles de bains, d'escrime et de danse dans l'aile est. Placés de part et d'autre de la cour, ces espaces sont éclairés par un saut-de-loup. Du côté de l'impasse Chartière, le mur de soutènement est recouvert de faïence blanche afin de réfléchir la lumière. Au rez-de-chaussée, un auvent en fer et en verre court le long de la cour. Au premier étage, de larges baies par groupe de trois sont encadrées de chambranles en brique de Bourgogne apparente; elles correspondent à des chambres d'élèves sur la rue Valette et à des salles d'études sur l'impasse Chartière. Le deuxième étage est en meulière hourdée à la chaux hydraulique ; une fenêtre sur trois est encadrée d'un fronton triangulaire avec en dessous, en mosaïque, le nom d'anciens élèves et membres du conseil d'administration de la société. Cet étage contient l'internat et le service de lingerie. Le troisième étage est identique au précédent, les mosaïques en moins ; il sert également à l'internat et à l'infirmerie. Le quatrième étage, mansardé, est couvert en ardoises et abrite des chambres pour certaines élèves et des logements de fonctions.

\section{PRINCIPALES PIÈCES}

Le réfectoire (ill. 4), pouvant contenir cinq cents couverts sur $600 \mathrm{~m}^{2}$, a été l'objet de soins particuliers, tant en matière d'hygiène que de décoration. 
III. 4 : « Réfectoire au collège Sainte-Barbe à Paris »

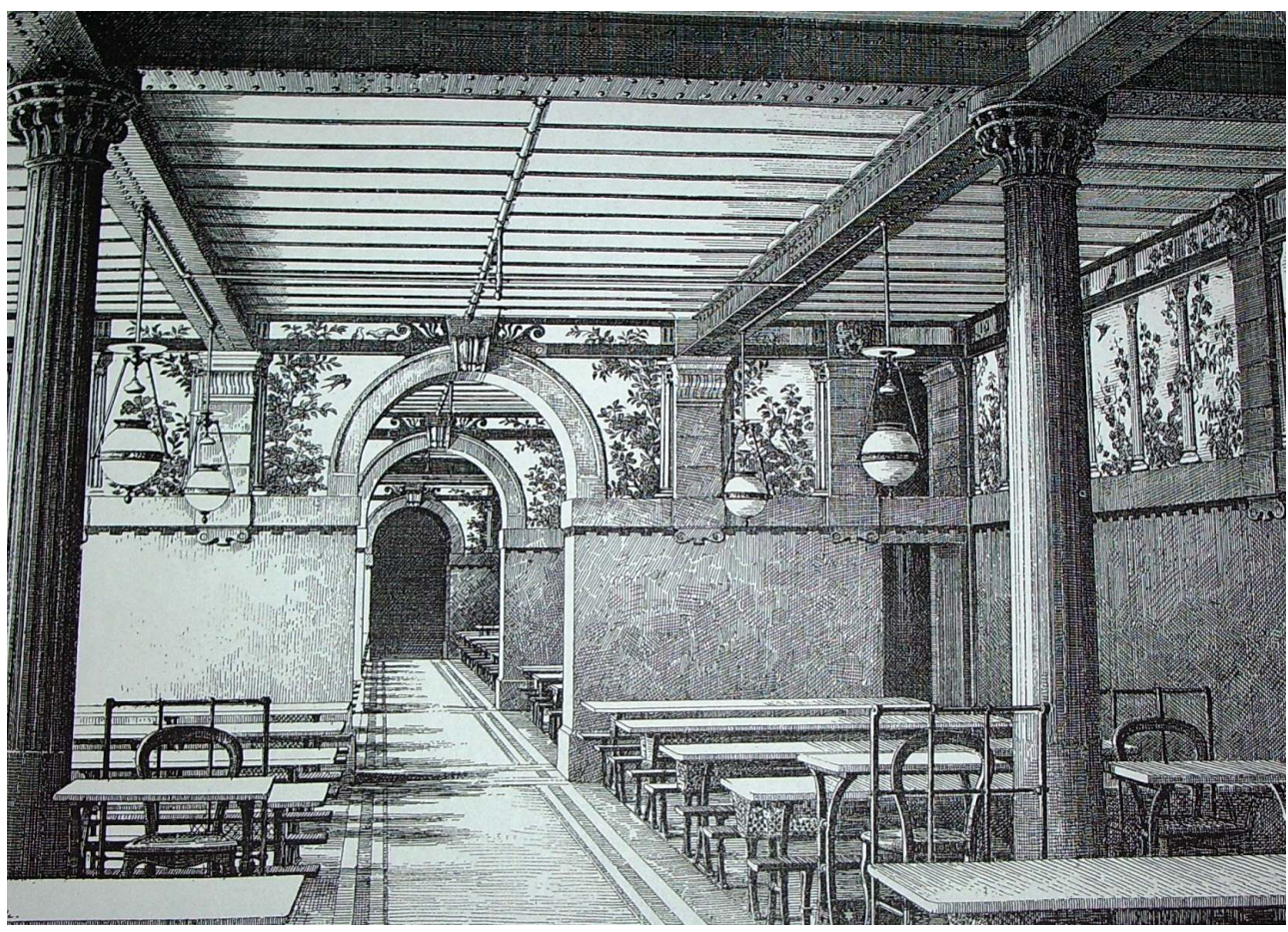

La Semaine des constructeurs, 28 février $1891,2^{e}$ série, $5^{e}$ année, p. 427

Cl. A. Lebarbé.

21 Il est divisé par des colonnes de fonte en trois grandes travées. Les tables sont alignées perpendiculairement au mur de la cour afin de faciliter la surveillance, et les deux travées le long du mur laissent un passage au surveillant; quatre personnes suffisent ainsi à surveiller toute la pièce. Le souci hygiéniste de pouvoir laver à grande eau toutes les parois a dicté le choix décoratif. Les murs sont recouverts de stuc cimenté sur environ deux mètres de haut, et dans leur partie supérieure par une mosaïque, exécutée par le mosaïste de l'Opéra de Paris, Facchina. Elle représente des feuillages et des oiseaux se détachant sur un fond bleu, qui rappellent la décoration des maisons de l'Antiquité et a valu au réfectoire son surnom de " pompéien ». Lheureux reprend ici le principe de décor imaginé par Labrouste dans le vestibule de la bibliothèque Sainte-Geneviève. Il donne l'illusion d'une "rangée de fenêtres ouvertes sur l'extérieur ${ }^{38}$, un artifice décoratif accentué par de fausses colonnettes cannelées qui supportent deux rangées de fausses poutres. Prévue pour cinquante élèves, la salle de dessin (ill. 5) mesure $200 \mathrm{~m}^{2}$. 


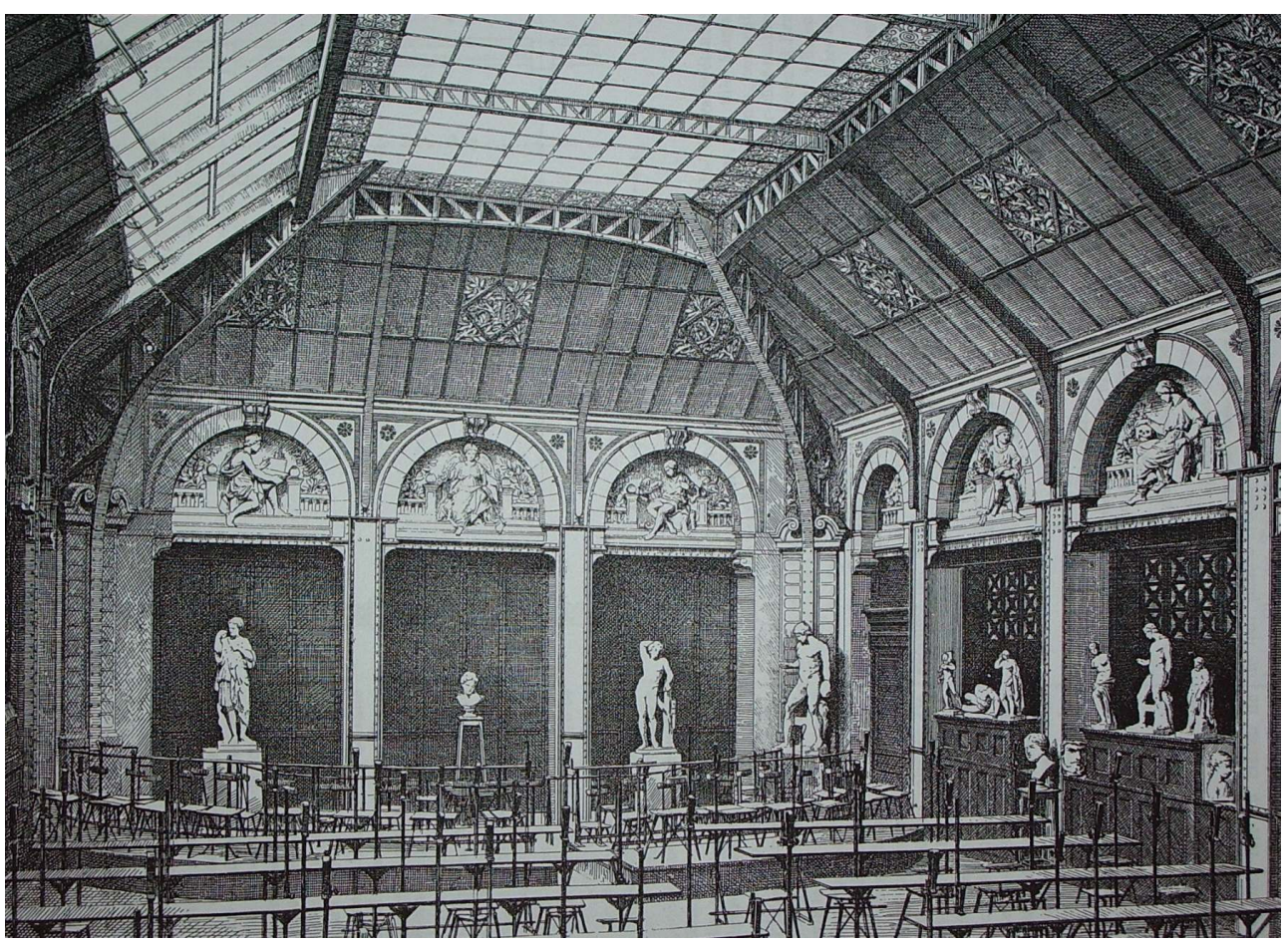

La Semaine des constructeurs, 21 février 1891, $2^{e}$ série, $5^{e}$ année, p. 415

Cl. A. Lebarbé

Construite entièrement en fer, elle ressemble beaucoup à la bibliothèque de l'École de droit, rue Cujas, que Lheureux vient d'achever (1876-1878). Au lieu de dissimuler le métal utilisé, auquel il est obligé d'avoir recours pour les grandes portées et les supports intérieurs, Lheureux rend visible " les fermes d'angles, les supports, les pannes, et toutes les pièces du plafond $»^{39}$ et fait un véritable travail de ferronnerie. Tous les côtés de la salle, excepté celui qui longe l'impasse Chartière, sont ornés de hauts-reliefs retraçant l'histoire du dessin depuis l'Antiquité. Les espaces qui séparent les poteaux métalliques ne sont fermés que par des cloisons en tôle, ce qui permet d'ouvrir la pièce sur les deux amphithéâtres voisins, et de disposer d'une très grande salle qui peut servir pour des cérémonies. Cette configuration avait déjà été mise en œuvre par Henri Labrouste en 1852 à Sainte-Barbe des Champs ${ }^{40}$ et par Eugène Train en 1865 au collège Chaptal.

Le grand amphithéâtre de chimie à gradins mobiles peut contenir jusqu'à cent vingt auditeurs; il est affecté à l'enseignement des sciences physiques et naturelles. Éclairé par une grande baie vitrée qui couvre toute la surface du mur de la rue Chartière à environ deux mètres du sol, il donne accès à un grand cabinet de physique qui renferme, dans des vitrines, tous les instruments utiles aux démonstrations.

L'internat occupe les deuxièmes et troisièmes étages des deux ailes du bâtiment de l'école préparatoire. Les lits en fer sont entourés d'une cloison de $1 \mathrm{~m} 60$ de haut, laissant au milieu du dortoir un passage. Chaque élève dispose ainsi d'une sorte de petite chambre, mais la surveillance reste facile grâce à la faible hauteur des cloisons. Un système de box individuels avec des demi-cloisons existe déjà à l'école Monge depuis 1878 ; la nouveauté réside dans l'agencement des lits qui ont été écartés des murs et des fenêtres afin d'éviter en hiver que la condensation humidifie les murs et les lits placés à côtét ${ }^{41}$. 
La réouverture de Sainte-Barbe en 1798 et son développement au XIX ${ }^{\mathrm{e}}$ siècle s'inscrivent dans une période d'organisation de l'enseignement, de développement de l'hygiène et d'évolution de la perception de l'enfant et de l'adolescent qui conduisent à penser de nouvelles formes architecturales adaptées et spécifiques, au moment même où s'élaborent des réponses architecturales nouvelles pour les hôpitaux et les prisons. Tandis que le Quartier latin est en pleins travaux, Sainte-Barbe réussit à étendre son emprise et à créer un complexe scolaire moderne dans son plan et ses matériaux. Cette œuvre, due aux architectes Théodore et Henri Labrouste puis à Ernest Lheureux, aboutit, malgré les nombreux problèmes, en particulier financiers, à laquelle l'institution est confrontée. Elle bénéficie du fort esprit de corps qui anime les anciens élèves qui dirigent l'institution, et qui leur a permis de trouver des réponses originales, comme la société en commandite puis la société anonyme, pour permettre son développement. Bénéficiant également de son statut particulier de société d'éducation non confessionnelle, dégagée des pesanteurs de la tutelle ministérielle, elle peut ainsi réaliser dans la pierre, très tôt dans l'histoire des constructions scolaires parisiennes, ses propres réponses architecturales à la question de l'enseignement.

\section{NOTES}

1. Le terme collège désigne le cursus scolaire des élèves qui se dirigent vers une formation longue. Le petit collège équivaut à l'école primaire et le moyen collège au collège actuel.

2. Jules Quicherat, Histoire de Sainte-Barbe: collège, communauté, institution, Paris, Hachette, 1860-1864, 3 vol.

3. Édouard Nouvel, Le Collège de Sainte Barbe. La vie d'un collège parisien de Charles VIII à nos jours, Paris, le collège Sainte-Barbe et l'association des anciens élèves, 1948.

4. Arch. de Paris, D $50 \mathrm{Z}$ 116, 121, 128, 130, 562 et 566.

5. Arch. de Paris, D 1 P 4/319 et 1094.

6. Arch. nat., archives de l'École des chartes non classées, "papiers d'érudits ", B 92. Outre la correspondance, il s'y trouve des notes d'Alexandre Labrouste intitulées Ma direction: souvenirs, notes pour mes mémoires, projets recommandés à mon successeur.

7. Encyclopédie d'architecture, 1884 ; Gazette de l'Instruction publique, Journal politique et littéraire, 20 décembre 1845 ; Le Génie civil, 30 août 1884 ; La Semaine du constructeur, 21 février et mars 1891.

8. Ancien prêtre théatin, Lanneau prête serment à la Constitution en 1791. Quatre ans plus tard, il est initié au Grand Orient de Paris et reste lié à la franc-maçonnerie. Arrêté sous la Convention, il est rapidement relâché et nommé directeur sous le directoire du Prytanée français. Il meurt en 1830.

9. Jacques Georgel, Anne-Marie Thorel, L'Enseignement privé en France (VII ${ }^{e}-\mathrm{XX}^{e}$ siècles), Paris, Dalloz, 1995, p. 22.

10. Édouard Nouvel, Le collège de Sainte Barbe. La vie d'un collège parisien de Charles VIII à nos jours, op. cit., p. 80.

11. Une société en commandite est composée de commanditaires; ils avancent des fonds aux commandités qui ont seuls le pouvoir de décision, contrairement à une société anonyme, dont les actionnaires ont un pouvoir de décision sur le conseil d'administration. 
12. Outre le plan de Quicherat reproduit ici, nous disposons, pour connaître les bâtiments avant leur reconstruction, de deux plans dans les feuilles d'immeubles du cadastre parisien (Arch. nat., $\mathrm{F}^{31} 71$, pièces 161 et 164 ).

13. Arch. de Paris, notes d'Alexandre Labrouste, D $50 \mathrm{Z} 116$.

14. Ibid.

15. Arch. de Paris, jugement du 19 juillet 1839, D 50 Z 116.

16. Discours d'Alexis Vavin lors du banquet du 4 décembre 1840, dans Association amicale des anciens élèves de Sainte-Barbe : Fêtes, comptes et rapports, Paris, E. Duverger, 1840, p. 12.

17. Les statuts de la société anonyme de Sainte-Barbe (11 juin 1840), l'ordonnance royale, et les autres actes concernant la société jusqu'en 1845 se trouvent dans les minutes du notaire Aubry (Arch. nat., Minutier central, XCIX/960).

18. Rapport anonyme aux anciens élèves, dans Association amicale des anciens élèves..., op. cit., p. 4.

19. Ibid.

20. Pour le règlement de leurs honoraires concernant l'aile sur la rue des Sept-Voies, la rue de Reims et la rue Jean Hubert reconstruite entre 1840 et 1842, les frères sont cités tous les deux au titre "d'architectes" (conseil d'administration du 18 février 1842, Arch. de Paris, D 50 Z 128). En revanche, pour la résolution des problèmes liés à l'alignement de la rue des Sept-Voies, à la réunion du 6 avril de la même année, seul Théodore est mentionné comme architecte (ibid.). Deux ans plus tard, pour l'édification $d u$ bâtiment rue Cujas, le compte rendu du conseil d'administration du 4 septembre 1844 spécifie que le directeur "a consenti avec M. Théodore Labrouste architecte du collège " (D $50 \mathrm{Z} 562$ ).

21. Abel Villemain, «Indication des locaux nécessaires pour l'établissement d'un collège royal », Bulletin universitaire contenant les ordonnances, règlements et arrêtés concernant l'instruction publique, 1843, n 41 , Paris, 1844.

22. Marc Le Cœur, «Les lycées dans la ville: l'exemple parisien (1802-1914)», Histoire de l'éducation, $\mathrm{n}^{\circ}$ 90, mai 2001, p. 148.

23. Rapport de Christian Dumas au conseil d'administration du collège, le 4 décembre 1843, dans Association amicale des anciens élèves..., op. cit., p. 19. L'entrée sur la rue Saint-Étienne-des-Grès est actuellement l'entrée de la bibliothèque de droit de la rue Cujas.

24. Notes d'Alexandre Labrouste à Jules Quicherat, Arch. nat., archives de l'École des chartes, B 92.

25. Ibid.

26. La bibliothèque Sainte-Geneviève est inaugurée le 4 février 1851.

27. Arch. nat., archives de l'École des chartes, B 92.

28. Révision cadastrale de 1876, rue Cujas, Arch. de Paris, D 1 P 4/319. Le cadastre ne concerne que l'aile qui s'ouvre sur la rue Cujas (qui suit le trajet de l'ancienne rue des Cholets) et celle qui longe la rue des Sept-Voies.

29. «Moi, je prie pour eux » (Jean 17, 9).

30. Notes d'Alexandre Labrouste, Arch. nat., archives de l'école des chartes, B 92.

31. "Voici mon commandement: aimez-vous les uns les autres comme je vous ai aimés » (Jean $13,34)$.

32. La rue du Four est perpendiculaire à la rue des Sept-Voies. La rue d'Écosse est perpendiculaire à la rue du Four et donc parallèle à la rue des Sept-Voies.

33. Discours de Bellaigue à l'assemblée générale des anciens élèves, dans Association amicale des anciens élèves..., op. cit., 1881, p. 16.

34. La rue des Sept-Voies est renommée rue Valette en 1879.

35. Eugène Varenne, Eugène Michel, «Enseignement : les grands établissements d'instruction, collège Sainte-Barbe à Paris ", Le Génie civil, n 18, 30 août 1884, p. 290.

36. Circulaires pour les commissions d'hygiène, 1865.

37. Ce gymnase a été démoli en 1930. 
38. Pierre Caspard, Jean-Luc Flohic (dir.), Le Patrimoine de l'éducation, Paris, Édition Flohic, Le patrimoine des institutions politiques et culturelles, 1999, p. 658.

39. Eugène Michel, Eugène Varenne, op. cit., p. 293.

40. Sainte-Barbe-des-Champs est situé à Fontenay-aux-Roses et accueille le petit collège de Sainte-Barbe (équivalent du primaire actuel). Premier collège à la campagne, Sainte-Barbe-desChamps est inauguré le 12 mai 1852.

41. Ibid., p. 294.

\section{RÉSUMÉS}

Ouvert en 1460 au coeur du quartier latin, contraint de fermer ses portes en 1793, le collège Sainte-Barbe renaît en 1798 grâce à Victor de Lanneau. Les directeurs successifs de l'établissement vont assurer son développement et lui donner des bâtiments nouveaux, avec la construction du collège en 1840 par les frères Labrouste, puis celle de l'école préparatoire en 1881-1884, par Ernest Lheureux. L'exemple de Sainte-Barbe se révèle particulièrement intéressant à une période où se définissent peu à peu une politique en faveur de l'enseignement secondaire et les réponses architecturales qui correspondent. Institution privée non confessionnelle, Sainte-Barbe dispose d'une grande liberté pour mettre en œuvre ses propres solutions innovantes en matière d'architecture scolaire, et bénéficie en outre du très fort esprit de corps de ses anciens élèves, qui lui permet de faire face aux difficultés financières. L'institution est ainsi l'une des premières à construire à Paris des bâtiments scolaires entièrement neufs et adaptés à leurs fonctions.

Opened in 1460 in the heart of the Quartier Latin, then forced to close down in 1793, the College Sainte-Barbe is brought back to life by Victor de Lanneau in 1798. The successive headmasters of the school will have it developped and enlarged with new buildings, first the construction of the secondary school by the Labrouste brothers in 1840 , then the one of the preparatory school by Ernest Lheureux in 1881-1884. The example of Sainte-Barbe turns to be of the utmost significance at a time when a policy in favour of secondary education and suitable architectural answers slowly take shape. As an undenominational private institution, Sainte-Barbe has full scope to carry out its own innovative designs as regards school architecture and is enabled to come up against financial difficulties thanks to the deep "esprit de corps" of its former pupils. Thus the institution is among the first ones to have proper brand new schools built.

1460 mitten im Quartier Latin gegründet, wurde das Collège Sainte-Barbe 1793 gezwungen die Türen zu schließen, und durfte erst 1840 auf Anregung von Victor de Lanneau einen neuen Schwung bekommen. Die aufeinanderfolgenden Schulleiter sorgten für die Entwicklung der Anstalt und statteten sie mit neuen Bauten aus: Der Collègebau wurde 1840 von den Gebrüdern Labrouste errichtet, der Studienkollegbau 1881-1884 von Ernest Lheureux. Das Collège Sainte-Barbe bietet ein treffendes Beispiel für die damalige Entwicklung einer Schulpolitik zugunsten der Sekundarstufe, die entsprechende architektonische Antworten verlangt. Das private aber nicht konfessionelle Institut Sainte-Barbe genießt weitgehende Freiheit, seine eigenen innovativen Schulbauprojekte durchzuführen und kann sich dabei auf den Korpsgeist seiner ehemaligen Schüler verlassen, um finanzielle Schwierigkeiten zu beseitigen. So zählt es zu den ersten Instituten, die in Paris völlig neue Schulbauten erstellten. 


\section{AUTEUR}

\section{ANNABELLE LEBARBÉ}

Annabelle Lebarbé a soutenu, sous la direction du professeur Jean-Noël Luc, une maîtrise d'histoire à l'Université de la Sorbonne - Paris IV en juin 2005 sur « L'espace de l'école : architecture et le mobilier du collège Sainte-Barbe de Paris 1798-1884 » Elle a soutenu l'année suivante un master 2 d'histoire sous la direction du professeur Jean-Pierre Chaline sur « L'aménagement du palais de l'Élysée sous la Ve République ». Adresse électronique : annabelle@lebarbe.net 